Rev. Elev. Méd. vét. Pays trop., 1973, 26 (2) : 203-20

\title{
La cysticercose musculaire des ruminants sauvages d'Afrique Centrale
}

\author{
par M. GRABER (*), P. M. TRONCY $\left({ }^{* *}\right)$ et J. THAL (**)
}

\begin{abstract}
RESUME
Les auteurs signalent, en République Centrafricaine, la présence de deux espèces de cysticerques dans les muscles et les organes de divers ruminants sauvages.

La première - la plus fréquente $(9 / 10$ des cas) - affecte le Buffle [Bubalus (Syncerus) caffer], le Cob de Buffon (Adenota kob), l'Hippotrague (Hippotragus equinus), ie Bubale rouge (Alcelaphus lelwel), l'Elan de Derby (Taurotragus derbianus) et le Guib harnaché (Tragelaphus scriptus). Elle représente la forme larvaire de Taenia hyaenae Baer, 1924; Verster, 1969 qui vit dans l'intestin de l'Hyène (Crocuta crocuta) et du Cynhyène (Lycaon pictus).

La seconde - plus rare (1/10 des cas) $\rightarrow$ n'a été rencontrée que chez le Buffle, le Bubale et l'Hippotrague. Le Cestode adulte correspondant est Taenia crocutae Mettrick et Beverley-Burton, 1961 de l'Hyène.

Le Buffle, le Cob de Buffon, le Guib hamaché et l'Elan de Derby sont des hôtes intermédiaires nouveaux de Taenia hyaenae et c'est la première fois que Taenia crocutae est identifié au nord de l'Equateur.

Les auteurs donnent des renseignements sur la fréquence et le niveau de l'infestation des bovidés autopsiés, ainsi que sur la localisation des parasites et la répartition de la maladie.

Ils envisagent les conséquences de cet état de choses sur l'utilisation des viandes de chasse pour la consommation humaine.
\end{abstract}

Les cysticercoses musculaires sont des cestodoses larvaires dues à la présence dans les muscles et dans divers organes de larves vésiculaires de type cysticerque qui, chez les ruminants sauvages de la grande faune aficaine, se présentent sous deux aspects :

I. - ILS SONT INERMES, sans rostre, ni crochets: il s'agit alors de Cysticercus bovis dont le Taenia adulte correspondant, Taenia saginata, vit dans l'intestin grêle de l'homme. Cysticercus bovis est surtout un parasite des Bovidés domestiques (zébu et bœuf).

(*) Laboratoire de Parasitologie, Ecole Nationale Vétérinaire, 2, quai Chauveau, 69337 Lyon, Cedex 1, France.

(**) I.E M.V.T., Laboratoire de Farcha, B.P. no 433, Fort-Lamy, Tchad.
Cependant, il est capable - mais beaucoup plus rarement - d'infester un certain nombre d'herbivores sauvages: Buffle [Bubalus (Syncerus) caffer] en Angola (48); Gazelle dorcas (Gazella dorcas), Gazelle corinne (Gazella rufifrons), Cob de Buffon (Adenota Kob) au Tchad (18, 19); Ourébi (Ourebia ourebi) en Zambie (23); Guib harnaché (Tragelaphus scriptus) et wildbeest (Connochaetes gnou) au Kenya (29); Girafes (Giraffa camelopardalis) venues d'Afrique et mortes dans divers jardins zoologiques de l'ancien et du nouveau monde $(11,38)$.

II. - ILS SONT ARMES. Dans ce cas, le scolex est pourvu d'un rostre et d'une double couronne de crochets alternants, inégaux et en forme de poignard. De tels cysticerques ont été, depuis une trentaine d'années, mis en évidence 
chez de nombreux Bovidés (17) appartenant:

- A la sous-famille des Antilopinae: Gazelle corinne; Gazelle de Grant (Gazella granti); Gazelle de Thomson (Gazella thomsoni); Guérénouk (Litocranius walleri); Impala (Aepyceros melampus).

- A la sous-famille des Reduncinae: Cob onctueù (Kobus défássa); "Lechwe (Onotragus leche); Cob de Buffon; Puku (Adenota vardoni); Reedbuck ou Cervicapre de roseaux (Redunca redunca); Ourébi.

- A la sous-famille des Madoquinae : Dikdik (Rhynchotragus kirki).

- A la sous-famille des Cephalophinae: Duiker ou Céphalophe couronné (Sylvicapra grimmia).

- A la sous-famille des Oryginae: Antilope rouanne (Hippotragus equinus); Antilope noire (Hippotragus niger); Oryx (Oryx gazella).

A la sous-famille des Alcelaphinae: Bubale rouge (Alcelaphus lelwel); Hartebeest (Alcelaphus buselaphus); Damalisque (Damaliscus korrigum); Sassaby (Damaliscus lunatus); Antilope gorgon ou Wildbeest.

- A la sous-famille des Tragelaphinae : Guib harnaché; Grand Koudou (Strepsiceros strepsiceros); Antilope canna ou Eland (Taurotragus oryx).

Les parasites ont été recueillis là où des enquêtes systématiques ont été effectuées, c'està-dire en Erythrée (12), au Kenya $(29,51)$, en Tanzanie (13, 44, 46), en Ouganda (21), en Zambie (23), en Rhodésie (39), en Afrique du Sud $(6,7,25,52)$ et au Tchad (19).

Ils ont été assimilés à plusieurs espèces : Cysticercus cellulosae, Cysticercus impalae Martinaglia et Brandt, 1947 (25) et, surtout, Cysticercus dromedarii, découvert par PELLEGRINI (32) dans les muscles et le toie des dromadaires (33), des Bovins (34) et des chèvres (36) de Somalie. Le Cestode adulte correspondant, Taenia hyaenae Baer, 1924 est un Taeniidae des Hyènes (Crocuta crocuta, l'Hyène tachetée; Hyaena Hyaena, l'Hyène rayée et Hyaena brunnea). Le cycle évolutif a été reproduit par PELLEGRINI $(35,37)$ en infestant d'une part des Hyènes avec des cysticerques de Dromadaire et, d'autre part, des bovins avec des anneaux gravides de Taenia hyaenae.
Ultérieurement, Cysticercus dromedarii que KUTZER et HINAIDY (22) ont mis en synonymie avec Cysticercus cameli, Nomani, 1920 (30) a été revu au Tchad $(18,20)$, en Ethiopie (10), au Soudan et en Egypte (16), tant chez des ruminants domestiques que chez des Antilopes.

Cependant, cette assimilation est loin de faire l'unanimité des auteurs. En 1961, METTRICK et BEVERLEY-BURTON (26) décrivent un nouveau parasite de l'Hyène, Taenia crocutae et, en 1969, Anna VERSTER (52) procède à une révision complète des Cestodes du genres Taenia, avec regroupement de plusieurs espèces. L'auteur rapporte (sans pouvoir - sauf pour Taenia hyaenae dont le cycle est connu - en donner la preuve formelle, puisque, pour ce faire, il faudrait pouvoir infester des ruminants avec des anneaux provenant de carnivores sauvages et réciproquement) les cysticerques armés des Bovidés sauvages africains à au moins quatre espèces de Taeniidae des carnivores: Taenia acinonyxi Ortlepp, 1938 du Guépard (Acinonyx jubatus) et de la Panthère (Panthera pardus); Taenia crocutae des Hyènes; Taenia gonyamai, Ortlepp, $1938 \mathrm{du}$ Lion (Panthera leo) et du Guépard et Taenia hyaenae de l'Hyène et du Cynhyène (Lycaon pictus).

L'étude d'une importante collection rassemblée par l'un d'entre nous (49) dans l'est de la République Centrafricaine a permis de confirmer partiellement le bien-fondé des observations faites en Afrique du Sud et d'éclairer certains aspects d'un problème particulièrement ardu.

\section{MATERIEL ET METHODE}

172 ruminants dont 97 Buffles et 75 Antilopes ont été autopsiés dans toute la zone cynégétique de l'est de la République Centrafricaine.

Une centaine de cysticerques ont été recueillis chez le Buffle, le Cob de Buffon, l'Antilope rouanne, Ie Bubale rouge, le Guib harnaché et l'Elan de Derby.

58 parasites (Buffle, 42; Cob de Buffon, 3; Bubale, 4; Elan de Derby, 3; Hippotrague, 4; Guib, 1) ont été ouverts et éclaircis au Lactophénol. Les scolex ont été placés entre lame et lamelle, légèrement écrasés, puis examinés au 


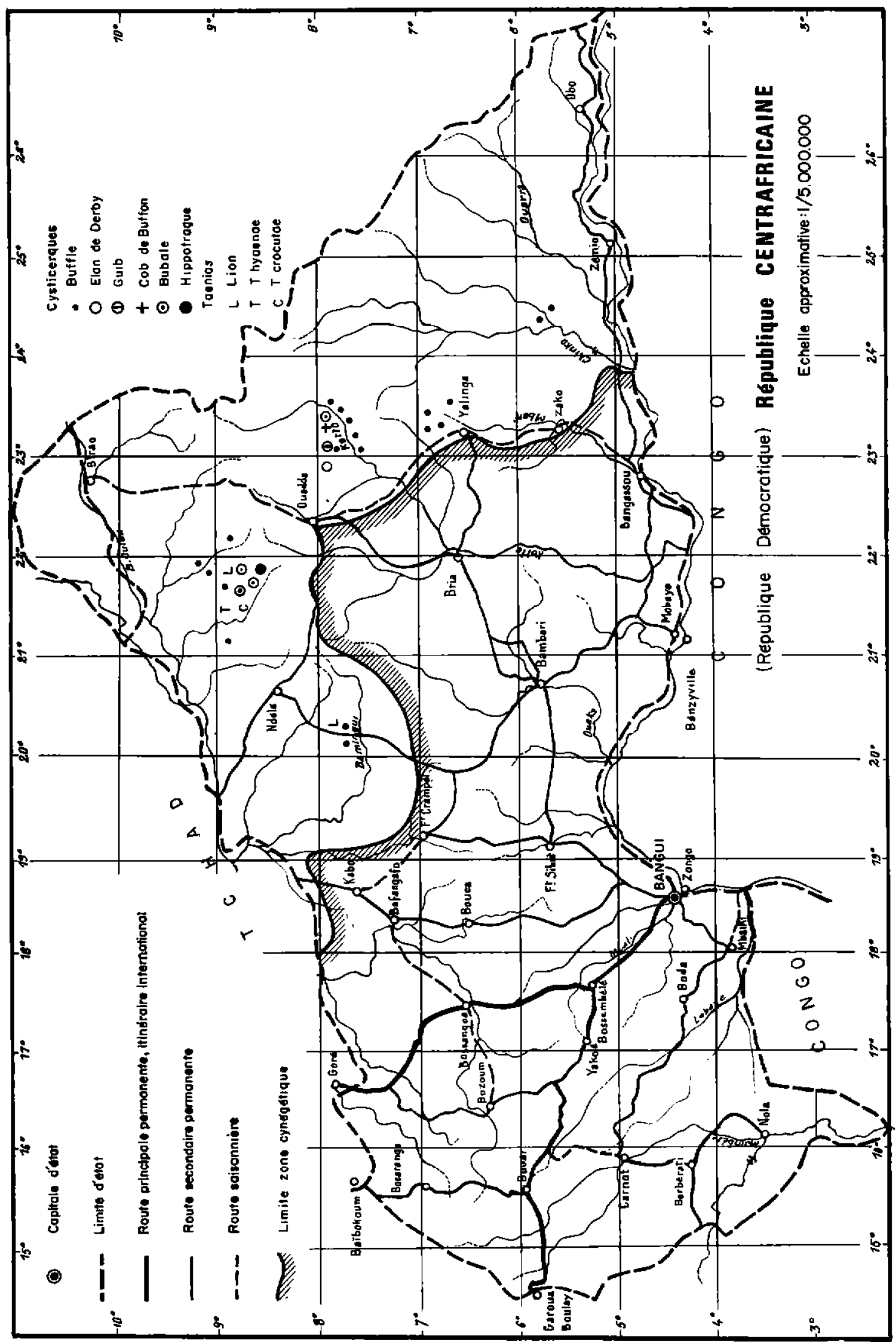


microscope. Les crochets ont été comptés, mesurés et dessinés.

Parallèlement, un certain nombre de Cestodes de Carnivores ont fait l'objet d'une étude précise. Il s'agit de :

- 12 Taenias de Lions abattus sur la Minia $\left(218 \mathrm{D} \mathrm{c}^{*}\right)$, la rivière Kapa $(218 \mathrm{D}$ d) et le Bamingui $(207 \mathrm{C} \mathrm{b})$.

- 16 Taenias prélevés dans l'intestin de deux Hyènes rayées tuées sur le Goro et la rivière Kapa (21 $8 \mathrm{D}$ d et $218 \mathrm{D} \mathrm{c})$.

Les segments de la chaîne ont été colorés au carmin chlorhydrique et les «têtes » ont subi le même traitement que celles des cysticerques.

Le scolex, le rostellum, les ventouses et les crochets des formes larvaires de ruminants sauvages et des Taenias adultes du Lion et de l'Hyène ont été systématiquement comparés.

\section{RESULTATS}

Deux espèces de cysticerques ont été rencontrées :

1. - La première - la plus fréquente ( $90 \mathrm{p}$. 100 des animaux parasités) - affecte le Buffle, le Bubale, le Cob de Buffon, l'Hippotrague, l'Elan de Derby et le Guib harnaché (tableau $\mathrm{n}^{\circ}$ II).

Les lésions ont l'aspect de kystes elliptiques ou subglobuleux mesurant 7 à $17 \mathrm{~mm}$ sur 4-9 mm (Pl. I, fig. 1 a). Les plus volumineux sont ceux que l'on trouve dans la langue $(14 \times$ $\left.6 \mathrm{~mm}^{* *}\right)$, le diaphragme $\left(13,7 \times 6,7 \mathrm{~mm}^{* *}\right)$ et le cour $\left(12,2 \times 6 \mathrm{~mm}^{* *}\right)$. Les kystes localisés dans les muscles du cou, des épaules et des membres postérieurs sont un peu plus petits (10-11 $\mathrm{mm} \times 5-6 \mathrm{~mm})$.

Ils sont entourés d'une mince membrane réactionnelle d'origine conjonctive.

Enchâssés dans la masse musculaire, ces kystes peuvent être facilement détachés: il persiste alors une alvéole qui marque l'emplacement du cysticerque (Pl. I, fig. $1 \mathrm{c}$ ).

L'ouverture du kyste permet de mettre en évidence le cysticerque proprement dit (Pl. I,

(*) Coordonnées I.B.A.H.

(**) Moyenne. fig. $1 \mathrm{~b})$ qui se présente sous la forme d'une vésicule remplie d'un liquide incolore dont l'invagination céphalique occupe presque toute la largeur. Placé dans de la bile à $39^{\circ} \mathrm{C}$, le parasite s'évagine et les détails anatomiques apparaissent mieux, notamment le scolex, le cou étroit et le début de la chaîne (Pl. I, fig. 2) qui est parfois très long (plus de $15 \mathrm{~mm}$ ). Le scolex (P1. I, fig. 2) porte quatre ventouses et un rostellum non rétractile armé d'une double couronne de crochets en forme de poignard, placés en alternance (Pl. I, fig. 3).

Les plus grands (Pl. II, fig. 1 et 2) sont pourvus d'un manche large, droit et plus long que la lame, ce qui leur donne un aspect caractéristique. La garde est, en général, entière, rarement mûriforme. Le bord postérieur présente, dans son tiers inférieur, une encoche, suivie d'un léger renflement.

Les plus petits (P1. II, fig. 1 et 2) ont le manche plus court que la lame qui est fortement courbée. La garde est bifide.

Quelques mensurations ont été effectuées : elles figurent au tableau $\mathbf{n}^{\circ} \mathbf{I}$.

A quel Taenia des carnivores sauvages correspond ce cysticerque?

a) Il ne s'agit certainement pas de Taenia acinonyxi du Guépard: cet animal, en effet, vit dans les zones subsahariennes ou sahéliennes et sa limite méridionale, dans le centre africain, est constituée par les $9^{\mathrm{e}}$ et $10^{\mathrm{e}}$ parallèles (24).

b) Taenia gonyamai dont la présence a été confirmée chez un Lion tué sur la rivière Kapa est caractérisé par un scolex un peu plus large $(1,3-1,4 \mathrm{~mm})$. Les crochets sont au nombre de 40 . Les plus grands mesurent en moyenne $192 \mu$ et les plus petits $136 \mu$, ce qui est conforme aux dimensions données par VERSTER (52) et par SACHS (43), mais absolument pas à celles du tableau $\mathrm{n}^{\circ} \mathrm{I}$.

c) Taenia crocutae - dont il sera question plus loin - est également différent : les crochets sont plus nombreux et leur taille plus réduite $(185 \mu$ pour ceux de la première rangée et $116 \mu$ pour ceux de la seconde).

d) Le seul Cestode qui se rapproche le plus des cysticerques de ce type est Taenia hyaenae décrit en 1923 (2) par BAER à partir de Cestodes récoltés dans l'intestin d'une Hyène 
TABLEAU $\mathrm{N}^{*} \mathrm{I}$

Mensurations des cysticerques de type I (Taenia hyaenae)

\begin{tabular}{|c|c|c|c|c|c|c|}
\hline & $\begin{array}{l}\text { Scolex } \\
\text { (en num) }\end{array}$ & $\begin{array}{c}\text { Rostellum } \\
\text { (en } \mu)\end{array}$ & $\begin{array}{l}\text { Ventouses } \\
\text { (en } \downarrow \text { ) }\end{array}$ & $\begin{array}{l}\text { Nombre de } \\
\text { crochets }\end{array}$ & $\begin{array}{l}\text { Longueur des } \\
\text { grands } \\
\text { crochets } \\
(\operatorname{en~} \mu)\end{array}$ & $\begin{array}{l}\text { Longueur des } \\
\text { petits } \\
\text { crochets } \\
(\text { en } \mu \text { ) }\end{array}$ \\
\hline $\begin{array}{l}\text { Buffle } \\
\text { Extrêmes } \\
\text { Moyenne (+) }\end{array}$ & $\begin{array}{c}0,7-1,3 \\
0,94 \pm 1,8 \\
(48++)\end{array}$ & $\begin{array}{c}300-500 \\
397 \pm 7.6 \\
(38++)\end{array}$ & $\begin{array}{c}300-440 x \\
200-350 \\
333 \pm 3,2 x \\
255 \pm 2,8 \\
(98++)\end{array}$ & $\begin{array}{l}32-39 \\
35 \pm 0,3 \\
(35++)\end{array}$ & $\begin{array}{l}204-236 \\
219 \pm 0,5 \\
(209++)\end{array}$ & $\begin{array}{l}128-160 \\
144 \pm 0.6 \\
(210++)\end{array}$ \\
\hline $\begin{array}{l}\text { Bubale } \\
\text { Extrêmes } \\
\text { Moyenne }(+++)\end{array}$ & $\begin{array}{l}0,8-1 \\
0,88\end{array}$ & $\begin{array}{c}400-440 \\
420\end{array}$ & $\begin{array}{l}320-360 x \\
240-300 \\
336,6 \times 256,6\end{array}$ & $31-35$ & $\begin{array}{l}204-232 \\
217,8 \\
(19++)\end{array}$ & $\begin{array}{l}128-156 \\
143,5 \\
(19++)\end{array}$ \\
\hline $\begin{array}{l}\text { Cob de Buffon } \\
\text { Extrêmes } \\
\text { Moyenne }(+++)\end{array}$ & $1-1,2$ & $460-570$ & & $32-33$ & $\begin{array}{c}2 O B-260 \\
230 \\
(7++)\end{array}$ & $\begin{array}{c}148-160 \\
153,7 \\
(7++)\end{array}$ \\
\hline $\begin{array}{l}\text { Hippotrague } \\
\text { Extrêmes } \\
\text { Moyenne }(+++)\end{array}$ & $\begin{array}{c}0,86-1,10 \\
0,99\end{array}$ & $\begin{array}{c}450-460 \\
455\end{array}$ & $\begin{array}{l}300-320 x \\
220-300 \\
309-244\end{array}$ & $\begin{array}{c}34-39 \\
37\end{array}$ & $\begin{array}{c}208-260 \\
232,6 \\
(12++)\end{array}$ & $\begin{array}{c}124-160 \\
145 \\
\left(15^{++}\right)\end{array}$ \\
\hline $\begin{array}{l}\text { Elan de Derby } \\
\text { Extrêmes } \\
\text { Moyenne }(+++)\end{array}$ & $\begin{array}{r}0,8-1 \\
0,83\end{array}$ & $\begin{array}{c}360-420 \\
(393)\end{array}$ & $\begin{array}{l}300-340 x \\
220-300 \\
305 \times 260\end{array}$ & $\begin{array}{c}35-39 \\
37\end{array}$ & $\begin{array}{c}212-22 B \\
219,2 \\
(18++)\end{array}$ & $\begin{array}{c}136-150 \\
144,2 \\
(14++)\end{array}$ \\
\hline $\begin{array}{l}\text { Guib } \\
\text { Extrêmes } \\
\text { Moyenne }(+++)\end{array}$ & 1,18 & 360 & $\begin{array}{l}360-400 x \\
240-280\end{array}$ & 33 & $\begin{array}{c}212-236 \\
228 \\
(6++)\end{array}$ & $\begin{array}{c}144-160 \\
151,2 \\
(5++)\end{array}$ \\
\hline $\begin{array}{l}\text { T. hyoence } \\
\text { (A. VERSTER, 52) } \\
\text { Extrêmes } \\
\text { Moyenne (+) }\end{array}$ & & & & $28-36$ & $\begin{array}{c}195-242 \\
217.7 \pm 11,9 \\
\left(49^{++)}\right.\end{array}$ & $\begin{array}{c}128-165 \\
146,9 \pm 9,5 \\
(41++)\end{array}$ \\
\hline
\end{tabular}

(+) Moyenne indiquée avec erreur type; (++) Nombre de données ; (+++) Moyenne sans erreur type.

tachetée abattue, au cours d'une expédition au Soudan inférieur, dans la région du Bahr-elZeruf. Le parasite a été revu depuis en Somalie (35), au Kenya (9), en Rhodésie (26), en Afrique du Sud (52) et au Tchad (50).

En R.C.A., les deux Hyènes abattues sur la rivière Kapa hébergeaient de nombreux Cestodes, en majorité Taenia hyaenae. Dans cette même zone, quatre Buffles et deux Bubales ont été trouvés porteurs de cysticerques étrojtement apparentés.

Taenia hyaenae mesure 15 à $35 \mathrm{~cm}$ de long sur une largeur maximale de $6,5 \mathrm{~mm}$. Le sco- lex (Pl. III, fig. 1), large de 0,9-1 mm porte quatre ventouses de $320-380 \mu \times 240-360 \mu$ et 'un rostellum de $360-400 \mu$ armé d'une double couronne de $32-36$ crochets dont la forme (P1 III, fig. 3) rappelle celle des crochets des cysticerques recueillis chez divers ruminants sauvages. Leur longueur varie de 204 à $222 \mu$ (en moyenne $217 \mu$ ) pour les plus grands et de 138 à $148 \mu$ (en moyenne $145 \mu$ ) pour les plus petits.

Au scolex, font suite le cou et le strobile, composé de segments dont les premiers sont plus larges que longs (Pl. III, fig. 2) et les der- 
niers plus longs que larges (de 5,5 à $6,5 \mathrm{~mm}$ sur 3,2 à $5 \mathrm{~mm}$ ).

Les pores génitaux sont irrégulièrement alternes et situés dans la moitié postérieure du segment (Pl. III, fig. 2).

Les testicules (plus de 320 ) sont répartis dans tout le parenchyme, en dedans des vaisseaux excréteurs, sauf au niveau des organes femelles. Ils sont plus nombreux du côté aporal. La poche du cirre est longue de $420-520 \mu$.

L'ovaire (1,9-2 mm) est bilobé, le lobe poral étant légèrement plus petit que le lobe antiporal (Pl. III, fig. 2). Le vitellogène, large de $1,4-1,5 \mathrm{~mm}$, est en arrière de l'ovaire. Le vagin qui est très rapproché de la poche du cirre est d'un sphincter puissant. L'atrium génital est large $(440-500 \mu$ sur $300-320 \mu$ ).

Dans les segments gravides (PI. V, fig. 3), l'utérus est constitué d'un axe longitudinal médian émettant 12 à 15 branches latérales plus ou moins ramifiées qui sont bourrées d'embryophores de 36-40 $\mu$ sur 28-35 $\mu$.

Le Cestode en cause correspond donc bien à Taenia hyaenae tel qu'il a été décrit par BAER (2, 3, 4), par METTRICK et BEVERLEY-BURTON (26) et par VERSTER (52).

En comparant les Cestodes adultes et les parasites larvaires, on est bien obligé de constater qu'il existe entre eux une grande ressemblance (aspect du scolex, forme et nombre de crochets). Par ailleurs, la longueur moyenne des grands et des petits crochets de Taenia hyaenae de République Centrafricaine et d'Afrique du Sud (52) d'une part, et des cysticerques du Buffle, du Bubale et de l'Elan de Derby d'autre part, est, à peu de choses près, la même.

En ce qui concerne les autres Antilopes du tableau $\mathrm{n}^{\text {10 }} \mathrm{I}$, si l'on adopte la nomenclature de VERSTER (52), on s'aperçoit que l'espèce Taenia hyaenae regroupe en réalité, outre ce parasite, un Cestode du Lycaon, précédemment décrit au Zaïre par BAER et FAIN (5) sous le nom de Taenia lycaontis. Dans ce cas, les crochets sont un peu plus longs que ceux de Taenia hyaenae: 212-242 (moyenne, $230 \mu$ ) pour ceux de la première rangée et 131-165 $\mu$ (moyenne, $152 \mu$ ) pour ceux de la seconde.

On retrouve ces dimensions chez les cysticérques du Cob de Buffon, de l'Hippotrague et du Guib (tableau no I), ainsi que chez deux Buffles.
La ladrerie des Bovidés sauvages semble donc provenir d'au moins deux sources différentes : l'hyène et le Lycaon, espèces qui sont toutes deux largement représentées dans l'Est de la République Centrafricaine.

2. - La seconde espèce est beaucoup plus rare que la précédente $(10$ p. 100 des cas $)$ et trois animaux seulement en étaient porteurs (tableau $\Pi^{\prime \prime}$ II) : un Bubale et un Hippotrague (Rivières Kapa-Vakaga - $218 \mathrm{D}$ c et $218 \mathrm{D} \mathrm{d}$ ); un Buffle dans le Bamingui (20 $7 \mathrm{C} \mathrm{b}$ ).

Les kystes sont, en général, de plus petite taille : $7-10 \times 3-4 \mathrm{~mm}$.

Le scolex (Pl. IV, fig. 1) qui a la même structure que celui de tous les Taeniidae mesure 0,8 à $1 \mathrm{~mm}$, les ventouses 300-340 $\mu \times 240$ $300 \mu$ et le rostellum $320-440 \mu$. Le nombre de crochets est compris entre 37 et 42 . Les plus grands (Pl. IV, fig. 2) ont une longueur de 176-200 $\mu$ (moyenne: $190 \mu$ ). La garde est épaisse, la lame fortement recourbée et le manche étroit. Comme dans le cas précédent, une encoche existe au tiers inférieur du bord postérieur.

Les crochets de la seconde rangée (P1. IV, fig. 3) sont plus petits : 104 à $128 \mu$ (moyenne : $116 \mu$ ). Le manche est droit, la garde bilobée ou cordiforme.

Si l'on se reporte à ce qui a été dit plus haut, ces cysticerques ne peuvent être assimilés, ni à Taenia gonyamai dont les petits crochets sont plus longs, ni à Taenia hyaenae, mais plutôt à Taenia crocutae, parasite qui infestait - en association avec d'autres Taeniidae — les deux Hyènes autopsiées sur la rivière Kapa, c'est-àdire à peu près au même endroit que le Bubale et l'Hippotrague porteurs de vésicules ladres.

Ce Cestode est très proche de Taenia hyaenae dont il ne diffère que par un certain nombre de caractères et de dimensions minimes, notamment le nombre de crochets $(38-40)$ et leur longueur : $192-200 \mu$ (moyenne : $192,5 \mu$ ) pour ceux du premier rang (Pl. V, fig. 1), 110-124 $\mu$ (moyenne : $117 \mu$ ) pour ceux du second ( $\mathrm{Pl}$. V, fig. 2). L'atrium génital est plus petit (300$320 \mu \times 200 \mu)$, ainsi que la poche du cirre $(300-340 \mu)$. Les lobes ovariens sont égaux et les branches utérines sont au nombre de 29-30 (P1. V, fig. 3). Les embryophores mesurent 36-40 $\mu$ sur $32-36 \mu$. 
TABLEAU $\mathrm{N}^{\circ} \mathrm{II}$

Cysticercose musculaire des Bovidês de R.C.A. - Taux d'infestation

\begin{tabular}{|c|c|c|c|c|c|c|c|}
\hline \multirow{3}{*}{ Es pẽ ce s } & \multicolumn{4}{|c|}{ Nombre d'Anima $\mathrm{a}$} & \multicolumn{3}{|c|}{ Taux d'infestation (p.100) } \\
\hline & \multirow{2}{*}{ Autopsiés } & \multicolumn{3}{|c|}{ Parasitês - Cysticerques } & \multirow{2}{*}{$\begin{array}{r}\text { Cysticerques } \\
T . \text { hyaenae }\end{array}$} & \multirow{2}{*}{$\begin{array}{l}\text { Cysticerques } \\
\text { T. crocutae }\end{array}$} & \multirow{2}{*}{ Tota1 } \\
\hline & & T. hyrenae & T. crooutae & Total & & & \\
\hline $\begin{array}{l}\text { Bovinae } \\
\text { Buffle }\end{array}$ & 97 & 17 & 1 & 18 & 18,5 & 1 & 19,5 \\
\hline $\begin{array}{l}\text { Roduncinae } \\
\text { Cob onctueux } \\
\text { Cob de Buffon } \\
\text { Cervicapre de roseaux } \\
\text { Ourébi } \\
\text { Total }\end{array}$ & $\begin{array}{r}13 \\
8 \\
2 \\
7 \\
30\end{array}$ & $\begin{array}{l}0 \\
1 \\
0 \\
0 \\
1\end{array}$ & $\begin{array}{l}0 \\
0 \\
0 \\
0 \\
0\end{array}$ & $\begin{array}{l}0 \\
1 \\
0 \\
0 \\
1\end{array}$ & 3,3 & 0 & 3,3 \\
\hline $\begin{array}{l}\text { Cephalophinae } \\
\text { Cêphalophe couronné }\end{array}$ & 3 & 0 & 0 & 0 & & & \\
\hline $\begin{array}{l}\text { Oryginae } \\
\text { Hippotrague }\end{array}$ & 10 & 0 & 1 & 1 & & 10 & 10 \\
\hline $\begin{array}{l}\text { Aloelaphinae } \\
\text { Bubale rouge } \\
\text { Damal isque } \\
\text { Total }\end{array}$ & $\begin{array}{r}23 \\
1 \\
24\end{array}$ & $\begin{array}{l}3 \\
0 \\
3\end{array}$ & $\begin{array}{l}1 \\
0 \\
1\end{array}$ & $\begin{array}{l}4 \\
0 \\
4\end{array}$ & $\begin{array}{l}13,1 \\
12,5\end{array}$ & $\begin{array}{l}4,2 \\
4,1\end{array}$ & $\begin{array}{l}17,3 \\
16,6\end{array}$ \\
\hline $\begin{array}{l}\text { Tragelaphinae } \\
\text { Elan de Derby } \\
\text { Guib harnaché } \\
\text { Total }\end{array}$ & $\begin{array}{l}5 \\
3 \\
8 \\
\end{array}$ & $\begin{array}{l}1 \\
1 \\
2\end{array}$ & $\begin{array}{l}0 \\
0 \\
0\end{array}$ & $\begin{array}{l}1 \\
1 \\
2\end{array}$ & 2,5 & 0 & 2,5 \\
\hline Total (sans buffles) & 75 & 6 & 2 & 8 & 8 & 2,6 & 10,6 \\
\hline Total (avec Buffles) & 172 & 23 & 3 & 26 & 13,3 & 1,8 & 25,1 \\
\hline
\end{tabular}

La comparaison entre Cestodes adultes et Cestodes larvaires laisse supposer qu'il s'agit bien là d'une seule et même espèce, Taenia crocutae. Les exemplaires de la République Centrafricaine présentent les mêmes caractères et les mêmes dimensions que ceux d'Afrique du Sud (50) et de Rhodésie (24).

Taenia crocutae est une espèce de création récente qui regroupe des Cestodes de Hyènes décrits précédemment sous le nom de Taenia hyaenae $(26,52)$. Sa présence a été signalée en Rhodésie, au Zaïre (Parc de l'Upemba), en Tanzanie $(9,46)$ et en Afrique du Sud.

C'est la première fois que l'on observe ce parasite en Afrique centrale.

\section{COMMENTAIRES}

1. En République Centrafricaine, les deux cysticerques identifiés peuvent être sûrement rapportés, d'une part à Taenia crocutae, et d'autre part à Taenia hyaenae, le second étant plus largement représenté que le premier.
Dans d'autres régions d'Afrique, notamment en Tanzanie (43, 46), en Afrique du Sud (52) et en Zambie, c'est l'inverse qui se produit : les cysticerques de type Taenia crocutae paraissent plus nombreux que ceux de type Taenia hyaenae.

Sont affectés :

- Par des cysticerques ayant pour origine Taenia crocutae : l'Impala, le Gnou, le Damalisque, le Bubale, le Waterbuck, le Dik-dik, l'Antilope rouanne, l'Antilope noire, l'Elan du Cap, la Gazelle de Thomson, la Gazelle de Grant, le Grand Koudou, le Lechwe et le Buffle;

- Par des cysticerques ayant pour origine Taenia hyaenae: l'Impala et l'Antilope noire en Afrique du Sud (52), le Damalisque, le Waterbuck, l'Antilope rouanne, la Gazelle corinne, le Bubale rouge, le Redunca et le Cephalophe couronné au Tchad (19). Le Buffle, l'Elan de Derby, le Cob de Buffon, le Guib harnaché sont donc des hôtes intermédiaires nouveaux de Taenia hyaenae. 
Les autres cysticerques sont moins bien connus. Ceux qui correspondent à Taenia acinonyxi n'ont été signalés qu'en Afrique du Sud (52). Les formes larvaires de Taenia gonyamai du Lion ont été rencontrées à plusieurs reprises chez l'Impala en Tanzanie (43) et, en Afrique du Sud, chez le Gnou, le Buffle et le Grand Koudou (52). En République Centratricaine, aucun cysticerque de ce type n’a pu être mis en évidence, alors que le Cestode adulte existe - mais en petit nombre - dans l'intestin du Lion (*).

2. Un autre point mérite de retenir l'attention. Les Hyènes - comme beaucoup de carnivores - sont susceptibles d'héberger plusieurs espèces de Taenias associés. C'est ce qu'avaient constaté DINNIK et SACHS (14) au parc de Serengeti en Tanzanie. C'est également vrai en République Centrafricaine. Dans l'intestin des deux Hyènes examinées, ont été isolés :

- dans le premier cas, Taenia hyaenae (six exemplaires) et Taenia crocutae (un exemplaire);

- dans le second, Taenia hyaenae (un exemplaire), Taenia crocutae (cinq exemplaires) et Taenia olngojinei Dinnik et Sachs, 1969 (trois exemplaires) $(* *)$.

Cette observation est intéressante. Elle semble confirmer l'hypothèse émise par VERSTER à propos de Cysticercus dromedarii (*). PELLEGRINI $(35,37)$ infeste des Hyènes à partir de cysticerques armés, prélevés chez des dromadaires et des bœufs. Il reproduit des Cestodes adultes. D'après la description de l'auteur, ceux-ci présentent des caractères communs à Taenia hyaenae et à Taenia crocutae. La taille des crochets (187 à $212 \mu$ et $112-137 \mu$ ) et leur nombre (36 à 46) recouvre ceux des deux espèces. La longueur de la poche du cirre est celle de Taenia hyaenae et le nombre de branches utérines (24-30) est celui de Taenia crocutaie. Il est probable que PELLEGRINI s'est trouvé en présence d'une infestation double par Taenia hyaenae et Taenia crocutae et que le nom de Cysticercus dromedarii ( $=$ cameli) correspond en réalité à deux espèces de Taen-

(*) Voir supra.

(*uk) Dont la forme larvaire vit dans l'espace épidural du sacrum de diverses Antilopes de l'Est africain. nias, voisines, mais différentes, susceptibles de cohabiter dans l'intestin du même hôte.

Chez les ruminants, les infestations mixtes sont possibles: c'est le cas d'un Hippotrague abattu au même endroit que la Hyène $n^{\circ} 1$ $(218 \mathrm{D} \mathrm{d})$. Dans les muscles de cet animal, ont été découverts un cysticerque de type Taenia crocutae et trois cysticerques de type Taenia hyaenae.

3. En République Centrafricaine, le taux d'infestation des 'Bovidés sauvages varie d'un animal à l'autre, comme l'indique le tableau $\mathrm{n}^{\circ}$ II. Les plus atteints sont dans l'ordre décroissant: le Buffle, le Bubale et l'Hippotrague.

Globalement, 15,1 p. 100 des ruminants examinés sont porteurs de cysticerques. En éliminant les Buffles, le pourcentage tombe à 10,6 p. 100 , c'est-à-dire du même ordre de grandeur qu'au Tchad (19).

Dans d'autres régions d'Afrique, des enquêtes menées sur une vaste échelle ont permis de préciser la fréquence de l'infestation, espèce par espèce. C'est ainsi qu'au Parc de Serengeti $(44,46), 80$ p. 100 des Gnous, des Bubales et des Gazelles de Grant, 71 p. 100 des Damalisques, 45 p. 100 des Impala, 30 p. 100 des Elans du Cap et des Waterbuck hébergent des cysticerques de diverses provenances (Taenia gonyamai et Taenia crocutae). Les Buffles sont moins parasités (18 p. 100).

En Afrique du Sud (53), la ladrerie due à Taenia gonyamai frappe un peu plus de 29 p. 100 des Buffles abattus.

Au Tchad (19), des cysticerques armés (surtout Taenia hyaenae) ont été observés chez la Gazelle corinne (10 p. 100), le Cervicapre de roseaux (12 p. 100), le Waterbuck (12 p. 100), le Bubale (15 p. 100), le Damalisque (plus de 30 p. 100), l'Hippotrague (25 p. 100) et le Céphalophe. Les neuf Buffles examinés étaient tous indemnes.

4. Le niveau de l'infestation est fonction du nombre de ruminants sauvages et de carnivores prédateurs qui sont en contact.

Le nombre de cysticerques rencontrés en R.C.A. est, le plus souvent, inférieur à 10. Toutefois, chez le Buffle, la cysticercose, mas- 
sive et généralisée est relativement fréquente : plus du tiers des animaux ladres.

Quant aux localisations, elles varient d'une espèce à l'autre. D'une façon générale, les cysticerques se voient principalement dans les muscles de la cuisse et de l'épaule, le cœur, le diaphragme et les muscles cervicaux. La langue et les masséters ne sont que faiblement infestés. Chez un Bubale et chez un Guib, des vésicules ladres ont également été découvertes dans le poumon.

En Tanzanie (46), la distribution des cysticerques dans l'organisme est quelque peu différente : pattes de derrière (100 p. 100), épaule (75 p. 100), muscles thoraciques (72 p. 100), muscles cervicaux (42 p. 100), masséters et cœur (40 p. 100), muscles abdominaux (22 p. $100)$.

En Afrique du Sud (6), les parasites se disséminent dans tous les muscles, avec une préférence marquée pour le cœur.

5. Au cours de cette enquête, cinq foyers ont pu être délimités :

- Celui de la moyenne Kotto (23 $7 \mathrm{C} \mathrm{d}\left({ }^{*}\right)$, 237 A c, 237 A d, 237 D c) : six Buffles, un Elan de Derby, un Guib, un Bubale et un Cob de Buffon;

- Celui de la rivière Chinko $(245 \mathrm{C} \mathrm{b})$ : deux buffles;

- Celui du Bamingui $(207 \mathrm{Cb})$ : deux Buffles;

- Celui des régions Yalinga - Mzali - Dji $(236 \mathrm{C} \mathrm{d}, 236 \mathrm{~B} \mathrm{c}, 236 \mathrm{C} \mathrm{c})$ : quatre Buffles;

- Celui des rivières Vakaga - Kapa (21 9 B d, 219 B b, 218 D c) : quatre Buffles, un Hippotrague et trois Bubales.

La cysticercose des Bovidés sauvages s'étend donc à l'ensemble de la zone cynégétique de $l^{\prime}$ Est centrafricain (Carte $\mathrm{n}^{*} \mathrm{I}$ ), du $5^{\circ}$ au $9^{\mathrm{e}}$ parallèle, c'est-à-dire du Zaïre au Tchad où la maladie est largement répandue dans tout le Salamat, notamment dans les zones d'Am-Timam et de Goz-Tougoula (19) qui marquent la frontière Nord-est de la République Centrafricaine.

6. La ladrerie des animaux de chasse pose dès à présent un grave problème d'hygiène pu-

(*) Coordonnées I.B.A.H. blique. En effet, dans un certain nombre de pays africains, il est de plus en plus question d'exploiter les potentialités de la faune sauvage et d'utiliser la viande et les abats des ruminants dans l'alimentation humaine (47). Or, ceux-ci hébergent souvent de nombreux parasites qui sont, la plupart du temps, transmis par des carnivores sauvages. C'est le cas non seulement des cysticerques des muscles ou des séreuses, mais encore des Sparganum, des Echinocoques, des Sarcosporidies et des Pentastomidés (Linguatula nuttalli).

Dans ces conditions, viandes et abats ne semblent pas dangereux pour la santé de l'homme (13), ce dernier n'intervenant pas directement dans le cycle évolutif. Mais, il n'en est pas toujours ainsi et des cas de ladrerie à Cysticercus bovis ont été signalés çà et là chez divers Bovidés sauvages d'Afrique.

En outre, les carcasses atteintes de cysticercose ont souvent un caractère répugnant. Pour ces deux raisons, les viandes ladres ne peuvent être livrées telles quelles à la consommation.

Après inspection sanitaire par un Vétérinaire, elles doivent être éliminées. La perte, d'après des estimations faites au parc de Serengeti (47) est d'environ 15-20 p. 100 de la valeur globale des carcasses, ce qui représente un lourd handicap dans les régions d'Afrique où la maladie sévit.

Les moyens de lutte sont, dans l'état actuel des choses, à peu près nuls. Il n'est guère concevable de déparasiter les Lions, les Hyènes et les Lycaon porteurs de Taenias. La seule solution consisterait à stériliser les viandes ladres par congélation rapide ou à les mettre en conserve. Malheureusement, ce n'est pas toujours possible, car les abattoirs susceptibles d'effectuer de telles opérations sont, généralement, fort éloignés des zones cynégétiques exploitables.

\section{CONCLUSIONS}

1. Les auteurs, dans l'est de la République Centrafricaine, signalent la présence de deux espèces de cysticerques, parasites des muscles des Bovidés sauvages:

- La première - qui est aussi la plus répandue ( $9 / 10$ des cas) — infeste le Buffle [Bubalus (Syncerus) caffer], le Cob de Buffon 
(Adenota Kob), l'Hippotrague (Hippotragus equinus), le Bubale rouge (Alcelaphus lelwel), l'Elan de Derby (Taurotragus derbianus) et le Guib harnaché (Tragelaphus scriptus).

Elle correspond à Taenia hyaenae Baer, 1924; Verster, 1969 qui vit dans l'intestin des Hyènes (Crocuta crocuta) et des Cynhyènes (Lycaon pictus).

- La seconde - beaucoup plus rare (10 p. 100 des cas) - affecte le Buffle, le Bubale et l'Hippotrague. Elle peut être rapportée à Taenia crocutae Mettrick et Beverley-Burton, 1961 de l'Hyène.

Les autres cysticerques musculaires, c'est-àdire ceux qui ressemblent à Taenia acinonyxi Ortlepp, $1938 \mathrm{du}$ Guépard (Acinonyx jubatus) ou à Taenia gonyamai Ortlepp, 1938 du Lion (Panthera leo) n'ont - pour l'instant - pas pu être mis en évidence.

2. Globalement, 15,1 p. 100 des ruminants sont porteurs des vésicules ladres. Les plus touchés sont dans l'ordre décroissant: le Buffle $(19,5$ p. 100$)$, le Bubale (17,3 p. 100$)$, l'Hippotrague (10 p. 100), le Cob de Buffon (1 sur 8 ), l'Elan de Derby (1 sur 5 ) et le Guib harnaché (1 sur 3 ).

Le Buffle, l'Elan de Derby, le Cob de Buffon et le Guib sont des hôtes intermédiaires nouveaux de Taenia hyaenae.

C'est la première fois que Taenia crocutae est identifié au nord de l'Equateur.
3. Le nombre de parasites rencontrés dépasse rarement le chiffre de 10 , sauf chez le Buffle où les cas de cysticercose généralisée sont nombreux (7,2 p. 100 des animaux autopsiés).

Les cysticerques se localisent dans les muscles de la cuisse, de l'épaule, le cour, le diaphragme et les muscles du cou. La langue et les masséters ne sont que faiblement atteints.

4. Les infestations mixtes de l'Hyène par Taenia hyaenae et Taenia crocutae paraissent fréquentes.

Chez l'hôte intermédiaire, les cysticerques correspondants sont quelquefois associés, notamment chez l'hippotrague.

5. La ladrerie des Bovidés sauvages pose un problème d'inspection sanitaire et risque de freiner les efforts faits dans différents pays d'Afrique pour mieux exploiter les possibilités alimentaires qu'offre la faune sauvage.

\section{Remerciements}

Les auteurs tiennent à remercier vivement Monsieur le Professeur Euzeby pour les facilités de travail qu'il leur a accordées dans son service de l'Ecole Nationale Vétérinaire de Lyon (France).

Nos remerciements iront également à Mademoiselle Lestra et à Monsieur le Dr Gevrey. 
Planche I. - Cysticerques du buffle ayant pour origine Taenia hyaenae.

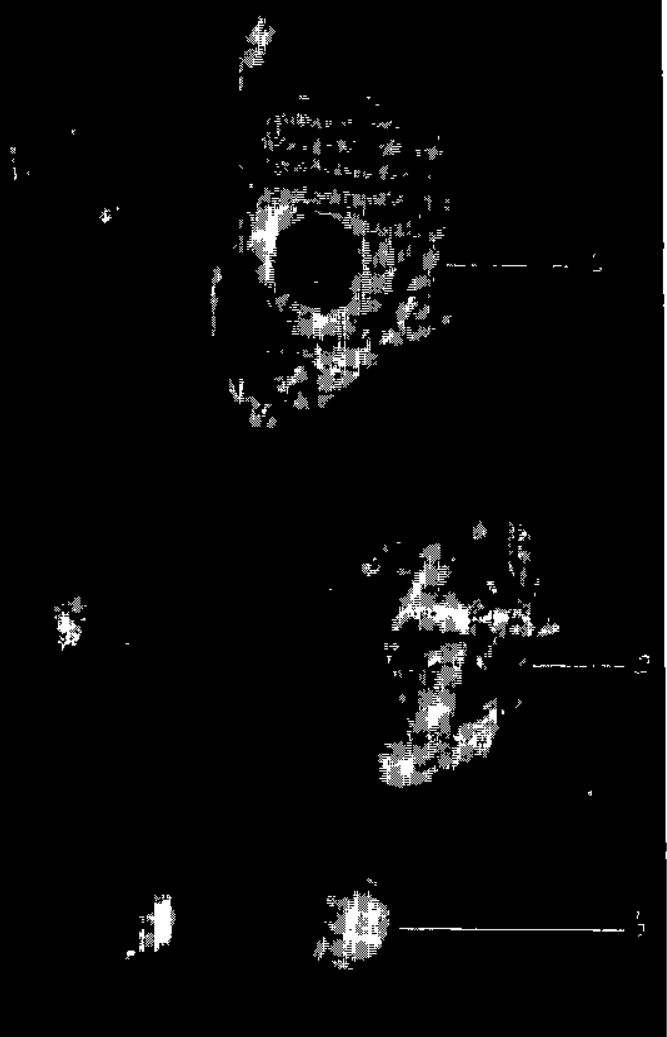

Fig. 1. - $a$ : le kyste; $b$ : cysticerques isolés; $c$ : alvéoles après énucléation du parasite.

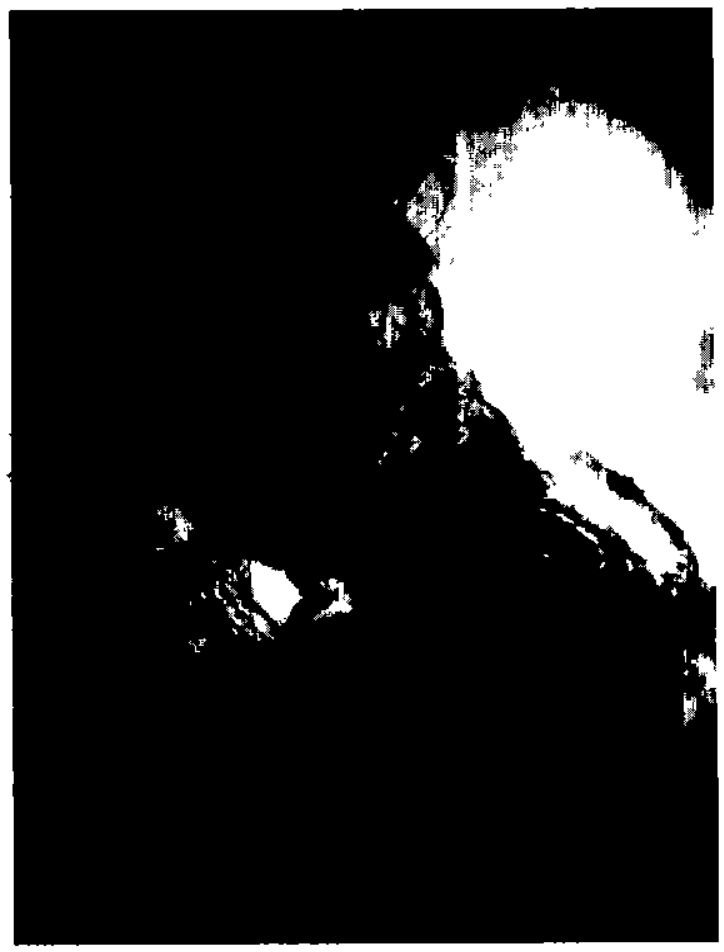

Fig. 2. - Scolex du cysticerque.

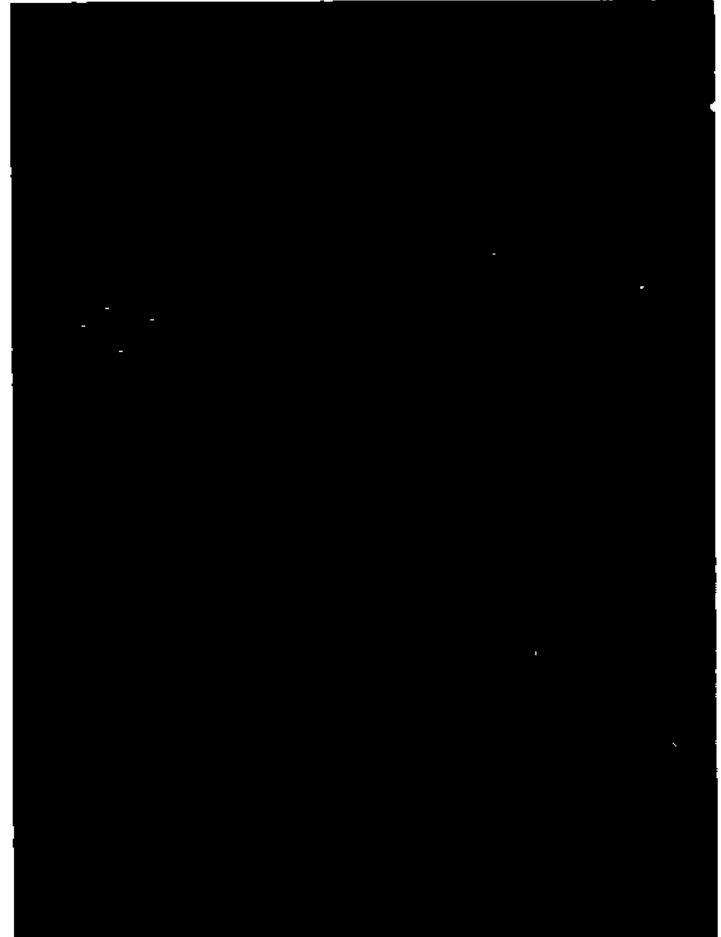

Fig. 3. - Les crochets. 
Planche II. - Cysticerques ayant pour origine Taenia hyaenae.

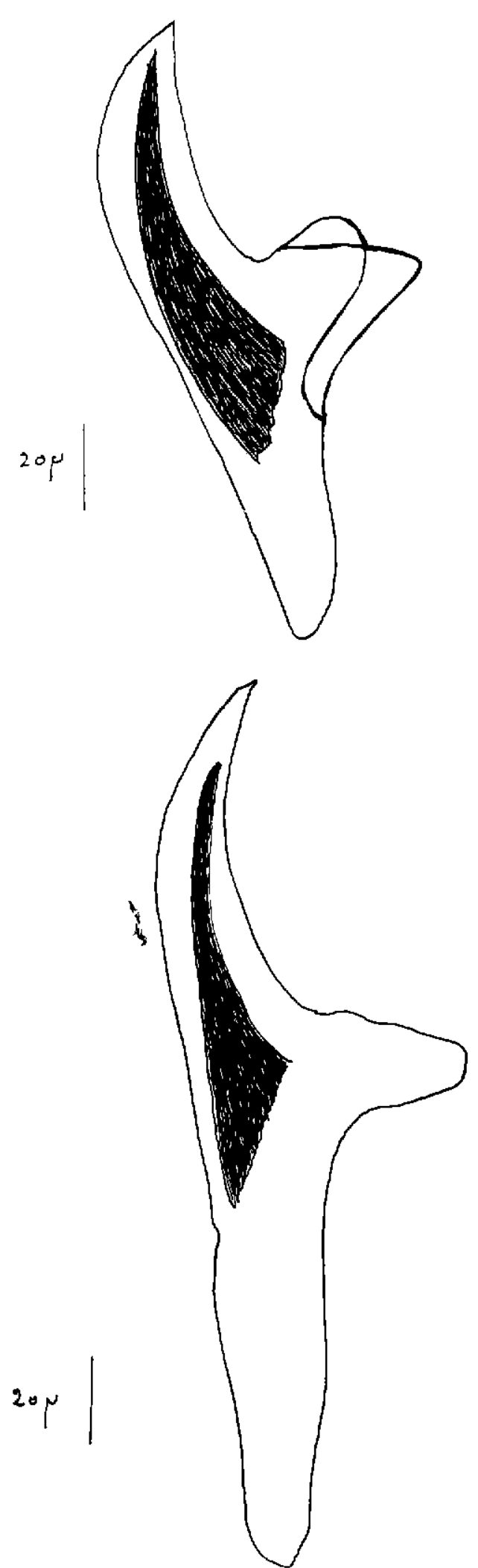

Fig. 1. - Grands et petits crochets de type Hyaenae «sensu stricto» (Bubale).
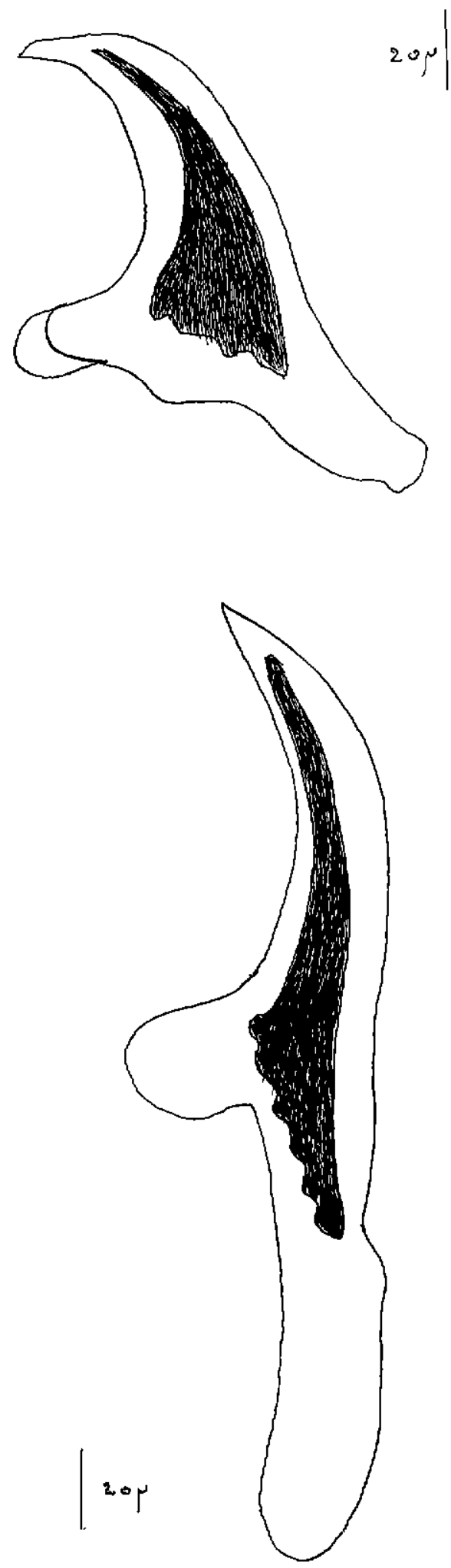

Fig. 2. - Grands et petits crochets de type lycaontis (Buffle). 
Planche III. - Taenia hyaenae de l'hyène.

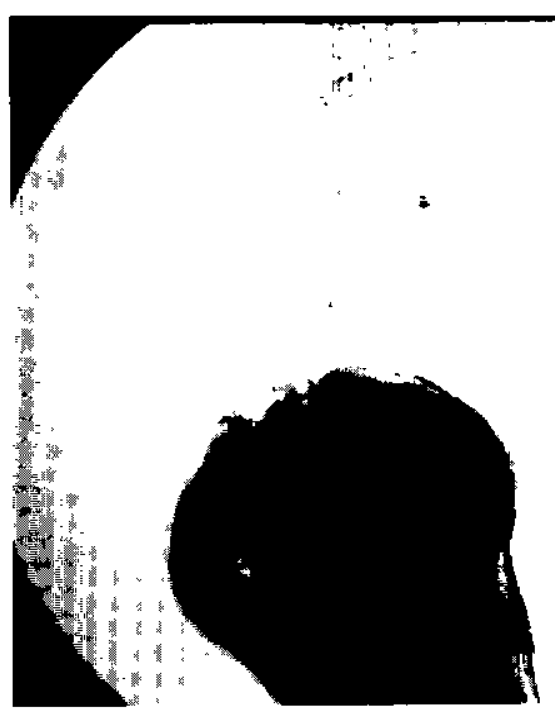

Fig. 1. - Scolex.

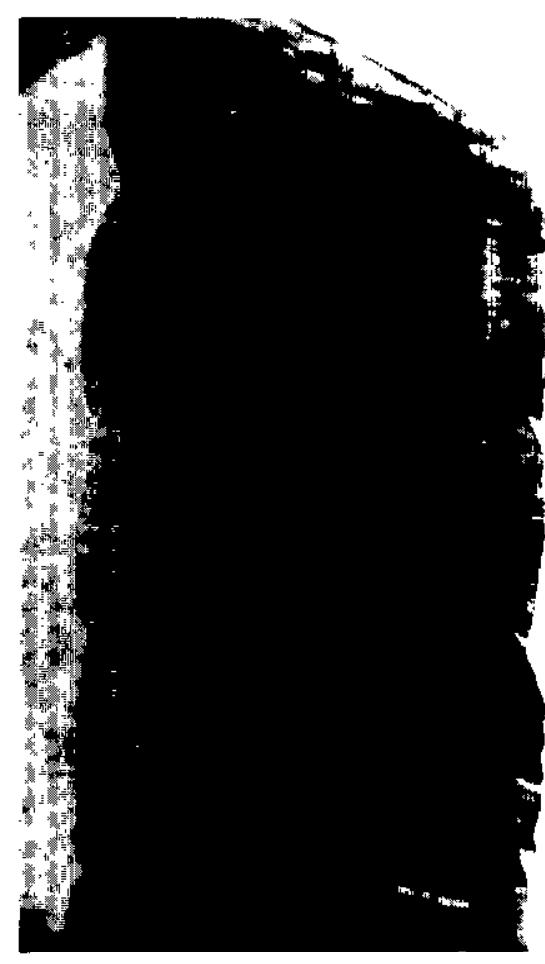

Fig. 2. - Anneau mûr.
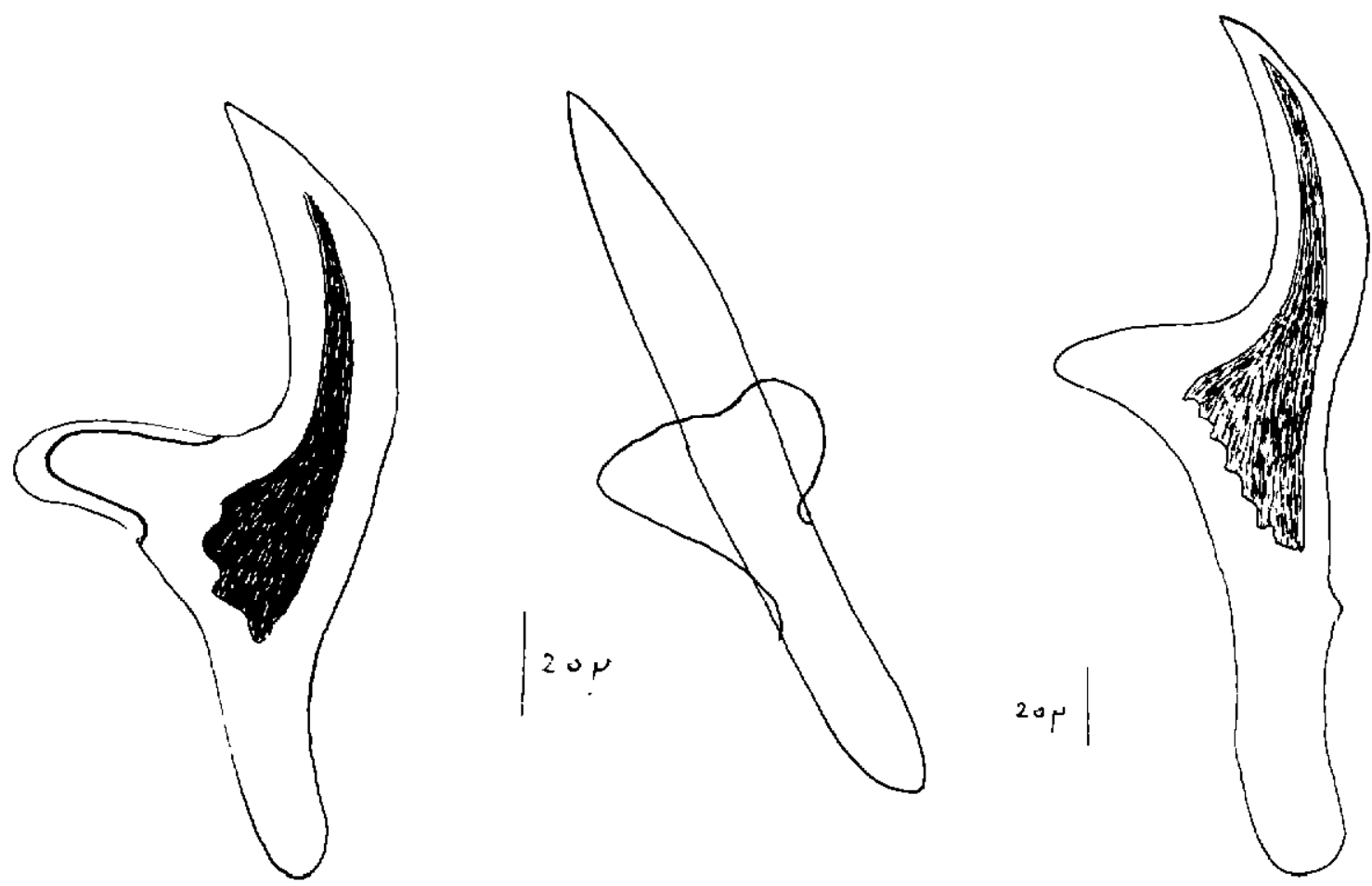

Fig. 3. - Grands et petits crochets. 
Planche IV. - Cysticerques du Buffle ayant pour origine Taenia crocutae.

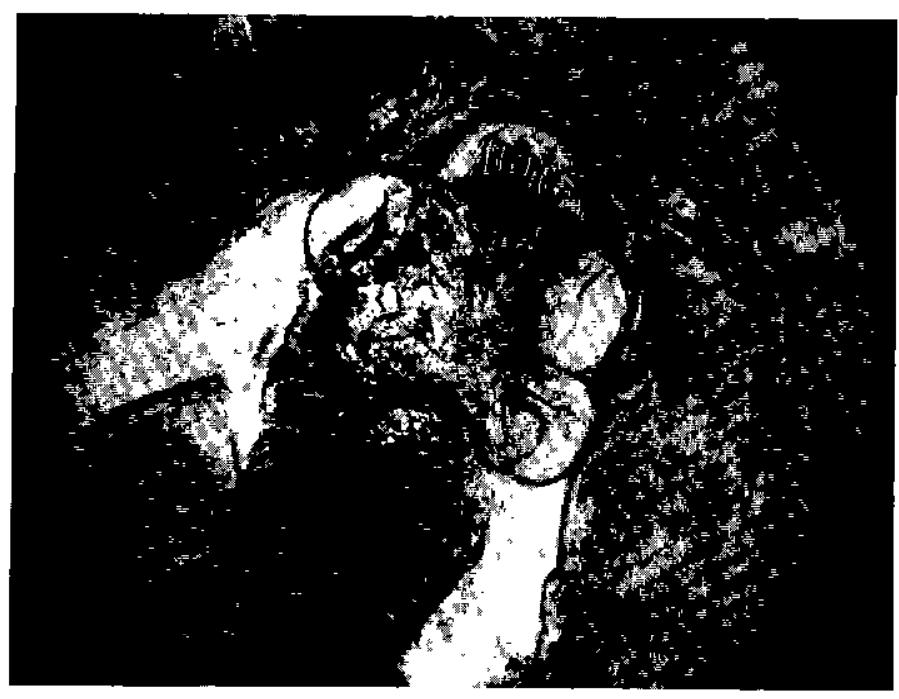

Fig. 1. - Scolex.

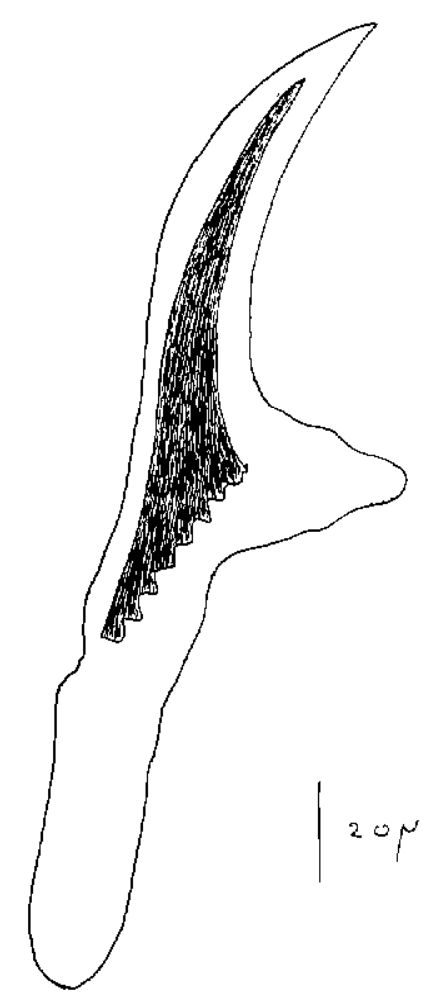

Fig. 2. - Grand crochet.

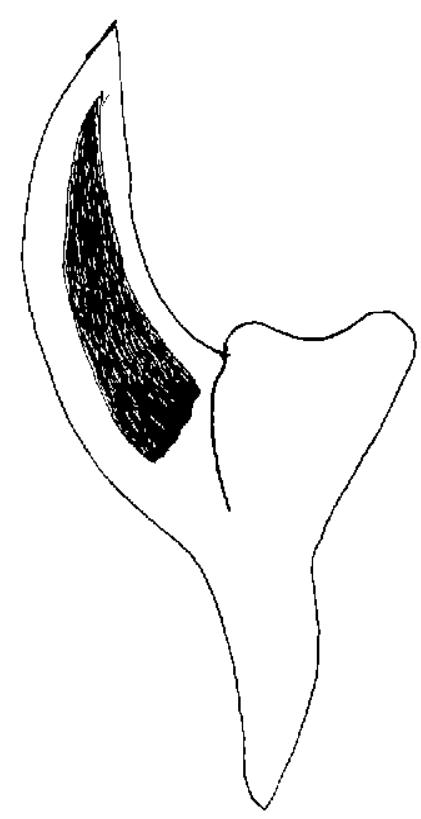

Fig. 3. - Petit crochet. 
Planche V. - Taenia crocutae de l'hyène.
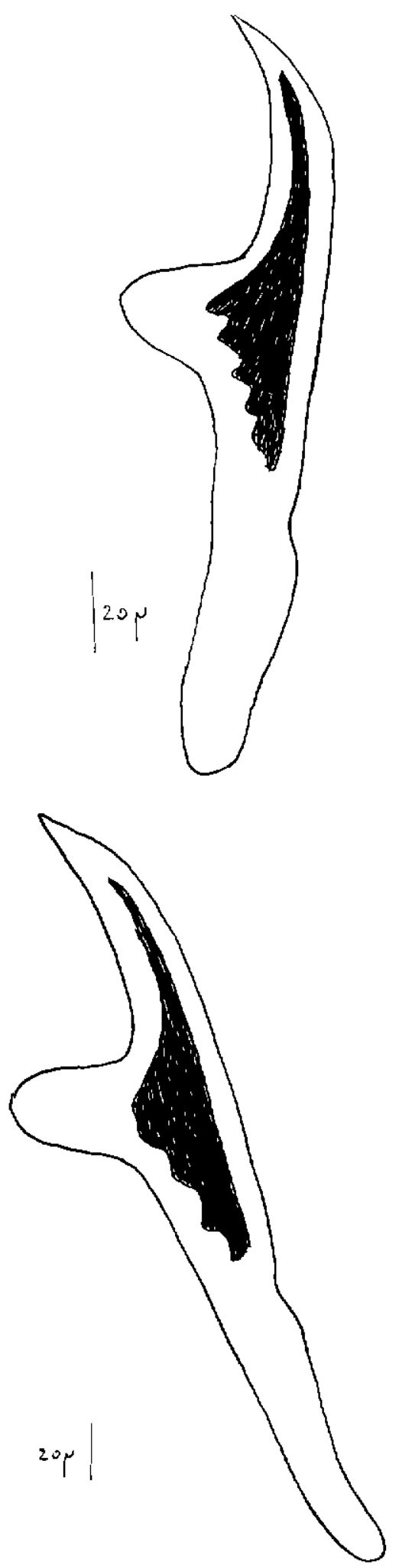

Fig. 1. - Grands crochets.
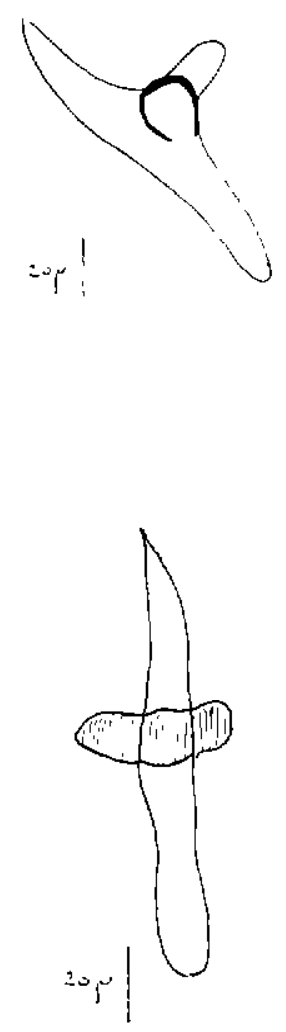

Fig. 2. - Petits crochets.

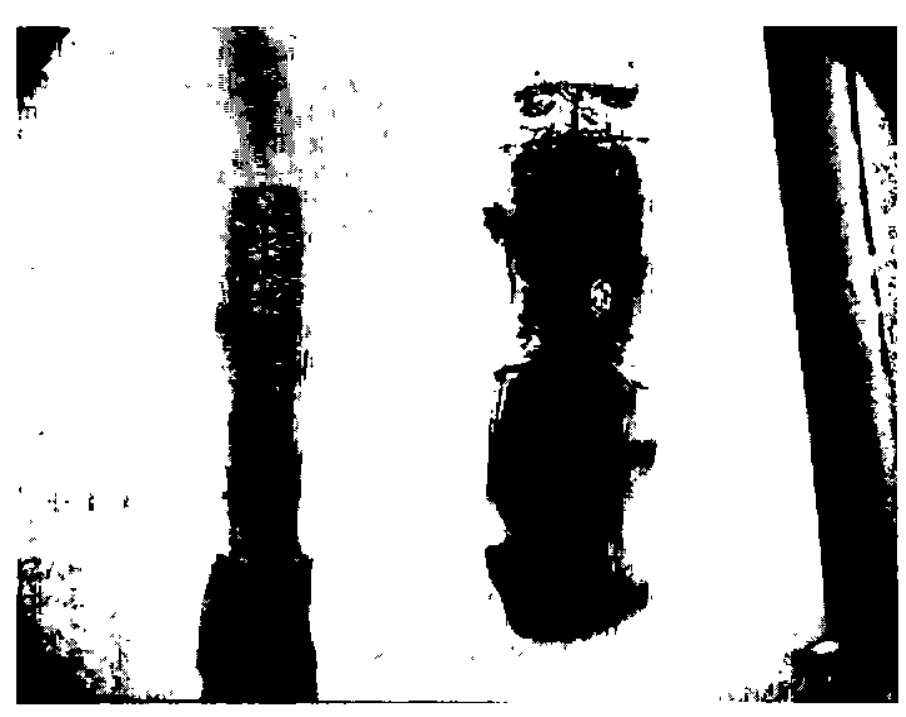

Fig. 3. - Anneaux gravides. A droite, Taenia hyaenae, à gauche Taenia crocutae. 


\section{SUMMARY}

\section{Muscular cysticercosis of Central African wild ruminants}

Investigations into cysticercosis of herbivorous game animals in the East of the Central African Republic indicates that the cysticerci recovered from the musculature and organs of wild ruminants are the larval stages of two Tapeworms species parasitizing predatory animals.

The former, Taenia hyaenae Baer, 1924; Verster, 1969, occurs in the spotted Hyaena (Crocuto crocula) and in the wild dog (Lycaon pictus); the latter, Taenia crocutae Mettrick et Beverley-Burton, 1961, is also a parasite of the spotted Hyaena.

Cysticerci of Taenia hyaenae have been recovered from african Buffalo (Bubalus (Syncerus) caffer), Kob (Adenota Kob), Hartebeest (Alcelaphus lelwel), Roan Antelope (Hippotragus equinus), Lord's Derby Eland (Taurotragus derbianus) and Bushbuck (Tragelaphus scriptus) and cysticerci of Taenia crocutae from african Buffalo, Hartebeest and Roan Antelope.

African Buffalo, Kob, Bushbuck and Lord's Derby Eland are new intermediate hosts of Taenia hyaenae.

Taenia crocutae is mentionned for the first time to the north of the Equator.

Taenia hyaenae and Taenia crocutae are often associated in the gut of their host and the cysticerci of both species in the flesh of some Antelopes.

The total incidence of muscular cysticercosis, the rate of infestation of various ruminants species, the geographical distribution, are recorded.

The authors emphasize consequences of cysticercosis which may have an adverse effect on the economic success of game cropping schemes, because parasited meat may be judged unsuitable for human consumption.

\section{RESUMEN}

\section{La cisticercosis muscular de los rumiantes salvajes de Africa Central}

Los autores señalan, en República Centroafricana, la presencia de dos especies de cisticercos en los musculos y los órganos de varios rumiantes salvajes.

La primera especie - la màs frecuente ( $9 / 10$ de los casos) - ataca el búfalo [Bubalus (Syncerus) caffer], el kobo de Buffon (Adenota Kob), el hipotrago (Hippotragus equinus), el búbalo rojo (Alcelaphus lelwel) la antilope de Derby (Taurotragus derbianus), y Tragelaphus scriptus. Esta especie representa la forma larval de Taenia hyaenae Baer, 1924; Verster, 1969 que vive en el intestino de la hiena (Crocuta crocuta) y de Lycaon pictus.

Se encontró la segunda - más escasa (1/10 de los casos) - sólo en el búfalo, el búbalo y el hipotrago. El Céstodo adulto correspondiente es Taenia crocutae Mettrick y Beverley-Burton, 1961 de la hiena.

El búfalo, el kobo de Buffon, Tragelaphus scriptus y la antilope de Derby son huespedes intermediarios nuevos de Taenia hyaenae y es la primera vez que se identifica Taenia crocutae en el norte del ecuador.

Los autores dan informes sobre la frecuencia y el nivel de infestación de los bovinos autopsiados, asi como sobre la localización de los parásitos y la repartición de la enfermedad.

Consideran las consecuencias de la cisticercosis sobre la utilización de carnes de caza para la alimentación humana.

\section{BIBLIOGRAPHIE}

1. ABULADSE (K. I.). Principles of cestodology (Ed. K.I. Skrjabin). Vol. IV. Taeniidae : Cestodes of animals and man and the diseases they provoke. Moscow Acad. Nauk. S.S.S.R., 1964 (en russe).

2. BAER (J. G.). Résultats zoologiques du voyage du Dr P.A. Chappuis au Nil supérieur. III. Helminthes. Revue suisse Zool., 1923, 30 (13): 344347.
3. BAER (J.G.). Contribution à la faune helmin. thologique sud-africaine. Annls. Parasit. hum. comp., 1924, 2 (3) : 239-247.

4. BAER (J.G.). Contributions to the helminth fauna of South Africa. 11-12 th Rep, Dir. vet. Educ. Res., Union S. Afr., 1926, 1: 105.

5. BAER (J. G.) et FAIN (A.). Cestodes. Exploration du parc national de l'Upemba. Mission 
G. F. de Witte. Inst. Parcs nat. Congo-Belge, Bruxelles, 1955, n* 36; 1-38.

6. BASSON (P. A.), Mc CULLY (R. M.), KRUGER (S. P.), VAN NIEKERK (J. W.), YOUNG (E.) et DE VOS (V.). Parasitic and other diseases of the african Buffalo in the Kruger national parc. Onderstepoort J. vet. Res., 1970, 37 (1) : 11-28.

7. BASSON (P. A.), Mc CULLY (R. M.), KRUGER (S. P.), VAN NIERKERK (J. W.), YOUNG (E.), KEEP (M. E.) et EBEDES (H.). Les epizooties du gibier en Afrique du Sud sur la base d'observations récentes. Inf. Méd, vét., 1971 (2/3) : 301328.

8. BATTELl (C.). Il C. dromedari (Pellegrini, 1945) in Eritrea. Boll. Soc. isal. Med. Ig. irop., $1949,9(3 / 4): 289-294$.

9. BAYLIS (H. A.). Records on some Helminths from the spotted Hyaena. Ann. Mag. nat. Hist., 1937,20 (4): 38-41.

10. BERGEON (P.). A veterinary parasitology survey. Report to the Government of Ethiopia. Rome, F.A.O., 1968, no T.A. 2458, 38 p.

11. BUCKLEY (J.J. C.). Cysticercus bovis in the liver of a Giraffe. Trans. R. Soc. trop. Med. Hyg., 1947, 41 (1) : 7.

12. CALL (C.). Il C. dromedarii (Pellegrini, 1945) in un Antilope Eritrea. Boll. Soc. ital. Med. Ig. trop., Sez Eritrea, 1949, 9 (3/4): 300-302.

13. DINNIK (J. A.) et SACHS (R.). Les cysticercoses, les echinococcoses et les sparganoses des herbivores sauvages d'Afrique orientale. Inf. Méd. vét., 1969 (2) : 108-118.

14. DINNIK (J. A.) et SACHS (R.). Zystizerkose der Kreuzbeinwirbel bei Antilopen und Taenia olngojinei sp. nov. der Tüpfelhyäne. Z. Parasitkde., 1969, 31 : 326-339.

15. EUZEBY (J.). Les maladies vermineuses des animaux domestiques et leurs incidences sur la pathologie humaine. Cestodes, T. II, fasc. I, Paris, Vigot Frères, 1966.

16. FAHMY (M. A. M.) et EL-AFIFI (A.). Cysticerci of the camel. Zentbl. Vet. Med., 1964, 11 (2) : 147-150.

17. FRECHKOP (S.) in: GRASSE (P.). Traité de zoologie. T. XVIl. Mammifères, fasc. 1, Paris, Masson et Cie, 1955.

18. GRABER (M.). La Cysticercose bovine. Son importance dans les zones sahéliennes d'élevage de la République du Tchad. Rev. Elè. Méd. vét. Pays trop., 1959, 12 (2): 121-148.

19. GRABER (M.). Pathologie de la faune sauvage du Tchad. Premiers résultats d'enquête. Rapport Ann. 1967, Laboratoire de Farcha, Fort-Lamy, Tchad, 1968, V: 110.

20. GRABER (M.) et CHAILLOUX (A.). Existence au Tchad de la ladrerie porcine à Cysticercus cellulosae. Rev. Elev. Méd. vét. Pays trop., 1970. 23 (1) : 49-55.

21. GUILBRIDE (P. D.), ROLLINSON (D. H. L.) et Mc AMULTY (E. G.). Tuberculosis in the free living African Cape Buffalo (Syncerus caffer Sparrmann). J. comp. Path. Ther, 1963, 73 (4) : 337-348.

22. KUTZER (E.) et HINAIDY (H. K.). Beitràge zur Helminthenfauna Egyptens. I. Cysticercus cameli Nomani, 1920. Zentbl Vet. Med., 1968, 15 B : 889-910.

23. LE ROUX (P.L.). The control of parasitic diseases in livestock. Rome, F.A.O., 1957, n $^{\circ} 696$, $20 \mathrm{p}$.

24. MALBRANT (R.). Faune du centre africain français. Paris, Lechevalier, 1952.

25. MARTINAGLIA (G.) et BRANDT (F. A.). Cysticercosis of the Impala or Rooibok (Aepyceros melampus) with reference to meat hygiene. J.S. Afr. vet. med. Ass., 1947, 18 (1) : 20-27.

26. METTRICK (D. F.) et BEVERLEY-BURTON (M.). Some Cyclophyllidean Cestodes from Carnivore in Southern Rhodesia. Parasitology, 1961, $51(3 / 4)$ : 533-544.

27. METTRICK (D. F.). Some Trematodes and Cestodes from mammals of Central Africa. Revta. Biol., Lisb., 1962, 3 (2/4) : 105.

28. MOHAN (R. N.) et GOTTS (M. G.). Diseases and parasites of the African Buffalo (Syncerus caffer). Vet. Bull., Weybridge, 1970, 40 (3) : 162.

29. NELSON (G. S.), PESTER (F. R. N.) and RICKMAN (R.). The significance of wild animals in the transmission of Cestodes of medical importance in Kenya. Trans, $R$. Soc. trop. Med. Hyg., 1965, 59 (5) : 507-524.

30. NOMANI (A.A.). A new armed hydatid in the Camel. Agric. J. Egypt., 1920, 10 : 69.

31. ORTLEPP (R. J.). South African Helminths. Part. II. Some Taenia of large wild carnivores. Onderstepoort J. vet. Res., 1938, 10 (2) : 253-278.

32. PELLEGRINI (D.). Cisticercosi del Cammello. Racc. Stud. Path. vet. Somaliland, 1942-45, 1 : 42-48.

33. PELLEGRINI (D.). Il «C. dromedarii n. sp. » nel Cammello e relativa Cisticercosi. Boll. Soc. ital. Med. Ig. trop., Sez Eritrea, 1947, 7 (3/4) : 317-324.

34. PELLEGRINI (D.). Il «C. dromedarii n. sp.» nel bovino. Boll. Soc. ital. Med. Ig. trop., Sez Eritrea, 1947, 7 (5/6) : 550-553.

35. PELleGRINI (D.). Il "C. dromedari " (Pellegrini, 1945) e lo stato larvale della Taenia hyaenae Baer, 1927. Boll. Soc. ital. Med. $1 \mathrm{~g}$. trop., Sez Eritrea, 1947, 7 (5/6): 554-565.

36. PELLEGRINI (D.). II «C. dromedarii $» \mathrm{n}, \mathrm{sp}$. nella capra. Boll. Soc. Ital. Med. Ig. trop., Sez Eritrea, 1949, 8 (3/4): 172-175.

37. PELLEGRINI (D.). Le Cysticercus dromedarii du Chameau et des Bovins (Pellegrini, 1945) et le «Taenia hyoenae * correspondant de l'hyène (Baer, 1927). Bull. Off. int. Epizoot., 1950, 33: 21-27.

38. PRICE (E. W.). A note on hepatic cysticercosis with the proposal of a new variety of Taenia saginata. J. Ala. Acad. Sct., 1961, 32 (4): 257261 .

39. ROTH (H. H.) et DALCHOW (W.). Untersuchungen iber den Wurmbefall von Antilopen in Rhodesien. Z. angew. Zool., 1967, 54 : 203-226.

40. ROUND (M. C.). Check-list of the helminth parasites of african mammals. Tech. comm. $n^{\circ} 38$. Common. Agric. Bur., 1968.

41. SACHS (R.). Note on cysticercosis in game animals of the Serengeti. $E$ Afr. wildl. J., 1966, 4 : 152-153.

42. SACHS (R.) et SACHS (C.). A survey of parasitic infestation of wild carnivores in the Serengeti region in northern Tanzania and the lake Rukwa region in southern Tanzania. Bull. epizoot. Dis. Afr., 1968,16 (4) : 455-472.

43. SACHS (R.). Untersuchungen zur artbestimmung und differenzierung der Muskelfinnen ostrafrikanischer Wiltiere. Z. Tropenmed. Parasis., 1969, 20 (1) : 39-50.

44. SACHS (R.). Uber den muskelfinnenbefall Wildbender Herbivoren des Serengetigebietes im Norden von Tanzania. Z. Jâgdwissench., 1969, 15 (4): 151-157.

45. SACHS (R.). Finnenbefall bei ostafrikanischen Antilopen und die verteilung der Muskelfinnen 
im Wildtierkörper, Fleischwirtschaft, 1969, 49 (10), 1331-1335.

46. SACHS (R.). Cysticercosis of East African game animals. J.S, afr. vet. med. Ass., 1970, 41 (2) : 79-85.

47. SCHINDLER (R.), SACHS (R.), HILTON (P.) et WATSON (R. M.). Some veterinary aspects of the utilization of African game animals. Bull. epizoot. Dis. Afr., 1969, 17 (2) : 215-221.

48. SOUSA DIAS (V.A.). Nota previa sobre os parasitas dos animais domesticos de Angola. Pecuaria. Ann. Serv. vet. Ind. Anim., Angola (1947-48), 1950, 2 : 53-59.

49. THAL (J.). Les maladies similaires à la peste bovine, étude et lutte, N'délé. Projet F.A.O.-
P.N.U.D., C.A.F. 13. Rapport Mission, I.E.M.V.T., Paris, 1972, 57 p.

50. TRONCY (P. M.). Rapport annuel. Laboratoire de Farcha (1969). Fort-Lamy, Tchad, 1970, 407.

51. URQUARTH (G. M.), HAY (D.), ZAPHIRO (D. R. P.) et SPINAGE (C. A.). Some internal parasites of game animals in Kenya. E. Afr. agric. For. J., 1960, 26 (1) : 11-20.

52. VERSTER (A.). A taxonomic revision of the Genus Taenia Linnaeus, 1758, s. str. Onderstepoort J. vet. Res., 1969,36 (1) : 3-58.

53. YOUNG (E.) et VAN DEN HEEVER (L. W.). The African Buffalo as a source of food and byproducts. J.S. Afr. vet. med. Ass., 1969, 40 (1): 83-88. 